CANCER

\title{
Molecular therapy for peritoneal dissemination of xenotransplanted human MKN-45 gastric cancer cells with adenovirus mediated Bax gene transfer
}

\author{
Y Tsunemitsu, S Kagawa, N Tokunaga, S Otani, T Umeoka, J A Roth, B Fang, N Tanaka, \\ T Fujiwara
}

Gut 2004;53:554-560. doi: 10.1136/gut.2003.021683

See end of article for authors' affiliations .....................

Correspondence to: Dr T Fujiwara, Division of Surgical Oncology, Department of Surgery, Okayama University Medicine and Dentistry, 2-5-1 Shikata-cho, Okayama 700-8558, Japan; toshi_f@ md.okayama-u.ac.jp

Accepted for publication 2 September 2003

\begin{abstract}
Background: Gene therapy is an innovative therapeutic approach for cancer. An adenoviral vector expressing the tumour suppressor p53 gene $(\mathrm{Ad} / \mathrm{p} 53)$ is currently under clinical evaluation for various cancers. We recently developed a binary adenoviral vector system that can express the strong proapoptotic gene Bax (Ad/PGK-GV16+Ad/GT-Bax: Ad/Bax).

Aims: To evaluate the potential of Bax gene therapy for gastric cancer, we assessed its antitumour effect in comparison with that of $p 53$.

Methods: The human gastric cancer cell lines MKN-1, MKN-7, MKN-28, and MKN-45 were treated with $\mathrm{Ad} / \mathrm{Bax}$ or $\mathrm{Ad} / \mathrm{p} 53$, and cell viability, transgene expression, and caspase activation were assessed in vitro. To compare the antitumour effects of $\mathrm{Ad} / \mathrm{Bax}$ and $\mathrm{Ad} / \mathrm{p} 53$ treatment in vivo, subcutaneous tumours and peritoneal dissemination of $M K N-45$ cells were generated in nude mice. Each mouse underwent intratumoral or intraperitoneal administration of viruses and the growth of implanted tumours was observed after treatment.

Results: Treatment with $\mathrm{Ad} / \mathrm{Bax}$ and $\mathrm{Ad} / \mathrm{p} 53$ resulted in marked Bax and p53 protein expression and effective apoptosis induction in MKN-1, MKN-7, and MKN-28 cells in vitro. In contrast, MKN-45 cells showed resistance to $\mathrm{Ad} / \mathrm{p} 53$ and only treatment with $\mathrm{Ad} / \mathrm{Bax}$ resulted in activation of caspase 3 expression and massive apoptosis. Ad/Bax treatment was more effective in suppressing both subcutaneous and peritoneally disseminated MKN- 45 tumours compared with $\mathrm{Ad} / \mathrm{p} 53$ treatment. Conclusion: $\mathrm{Ad} / \mathrm{Bax}$ treatment significantly inhibited the growth of even $\mathrm{p} 53$ resistant gastric cancer in vitro and in vivo. Therefore, adenovirus mediated Bax gene transfer may be useful in gene therapy for gastric cancers.
\end{abstract}

G astric cancer is one of the most common malignancies in the world, especially in Eastern Asia. Although in its early stages the disease is treatable with endoscopic or surgical resection, advanced gastric cancer does not generally respond to conventional chemotherapy or radiotherapy. ${ }^{1}$ In view of the fact that the antitumour effect of most chemotherapeutics is mediated through induction of apoptosis, refractoriness of gastric cancer may be due to its non-susceptibility to apoptosis. Therefore, altering the threshold of apoptosis in this cancer would be a rationale strategy. Gene therapy is being established as a promising new modality based on emerging knowledge in molecular biology, and it is an attractive alternative for treatment of gastric cancer.

Clinically, p53 is one of the most potent genes in gene therapy for cancer. ${ }^{2}{ }^{3}$ Activation of $p 53$ leads to either Gl arrest through induction of $p 21$ expression or apoptosis, in part by activation of Bax expression. ${ }^{4}$ Introduction of the $p 53$ gene via a recombinant adenovirus has been shown to inhibit the growth of various cancer cells in vitro and in vivo, including gastric cancer cells. ${ }^{5-11}$ However, other previous reports showed that cancer cells expressing wild-type $p 53$ are relatively resistant to treatment with the $p 53$ gene; thus they may not be appropriate targets for this gene. ${ }^{12}{ }^{13}$ In fact, $p 53$ gene transfer was insufficient for controlling the growth of gastric tumours with cells expressing wild-type $p 53 .^{10}$

Bax is a strong proapoptotic gene that causes cytochrome c release from mitochondria, activates the caspase pathway, and leads to apoptosis. ${ }^{14}{ }^{15}$ We developed a binary adenoviral vector system for Bax overexpression. ${ }^{16}$ Our system can transfer the Bax gene and induce apoptosis in vivo. Therefore, in this study, we examined the therapeutic potential of the Bax gene in gastric cancer cell lines, as a more effective strategy than use of the $p 53$ gene. We clearly show that adenovirus mediated Bax gene transfer is a more potent gene therapy modality for gastric cancer in both an in vivo subcutaneous tumour model and intraperitoneal dissemination model.

\section{METHODS}

Cell lines and adenoviruses

The human gastric cancer cell lines MKN-1, MKN-7, MKN28, and MKN-45 were grown in RPMI 1640 medium supplemented with $10 \%$ fetal bovine serum and antibiotics. MKN-1, MKN-7, and MKN-28 are homozygous for a mutation in $p 53$ at codon 143 (Val to Ala), 278 (Pro to Ser), and 251 (Ile to Leu), respectively. MKN-45 contains wild-type p53. The adenoviral vectors used in this study were the following: recombinant adenoviruses regulated by the GAL 4/

\footnotetext{
Abbreviations: GT, GAL4/TATA; GV16, GAL4/VP16 fusion protein; PGK, 3-phosphoglycerate kinase; CMV, cytomegalovirus; vp, viral particles; pfu, plaque forming units; $\mathrm{MOI}$, multiplicity of infection; $X$-gal, 5-bromo-4-chloro-3-indolyl- $\beta$-D-galactopyranoside; XTT, 2,3-bis-(2methoxy-4-nitro-5-sulphenyl)-(2H)-tetrazolium-5-carboxanilide; PBS, phosphate buffered saline; SDS, sodium dodecyl sulphate; PAGE, polyacrylamide gel electrophoresis
} 
TATA (GT) minimal synthetic promoter and containing human Bax- $\alpha$ gene cDNA (Ad/GT-Bax) or Escherichia coli $\beta$-galactosidase gene (Ad/GT-LacZ); the GAL4/VP16 fusion protein (GV16) transactivating protein for the GT promoter under the control of the 3-phosphoglycerate kinase (PGK) promoter (Ad/PGK-GVl6); and human wild-type $p 53$ gene regulated by the cytomegalovirus (CMV) promoter enhancer (Ad/CMV-p53; namely Ad/p53). ${ }^{16}$ All of the viruses were propagated in a package containing 293 cells, purified twice via ultracentrifugation in a cesium chloride gradient, and subjected to dialysis. The titre for each virus vector was determined according to the absorbency of the dissociated virus at $\mathrm{A}_{260 \mathrm{~nm}}$ and using the plaque assay. ${ }^{18}$ Each titre described in this report is expressed in viral particles (vp) and was determined according to $\mathrm{OD}_{260 \mathrm{~nm}}$ rather than plaque forming units (pfu). The vp:pfu titre ratio for the vectors used in the study was 30-100:1. In a binary vector system, expression of the Bax gene can be induced by transferring Ad/ GT-LacZ or Ad/GT-Bax into target cells along with Ad/PGKGV16. For this purpose Ad/GT-LacZ or Ad/GT-Bax was mixed with Ad/PGK-GVl6 at a ration of 1:1 (namely Ad/LacZ and $\mathrm{Ad} / \mathrm{Bax}$, respectively).

\section{$\beta$-galactosidase expression}

Cells were plated in 24 well culture plates at a density of $3 \times 10^{4} \mathrm{cell} /$ well and infected with $\mathrm{Ad} / \mathrm{LacZ}$ at a multiplicity of infection (MOI, vp/cell) ranging from 0 to 7500 . After 48 hours, 5-bromo-4-chloro-3-indolyl- $\beta$-D-galactopyranoside (X-gal) was used as a substrate to evaluate $\beta$-D-galactosidase expression, which was indicated by a blue precipitate in the cell.

\section{Assay of cell viability}

Cells were plated in 96 well plates at a density of 5000 cells/ well and infected with Ad/LacZ, Ad/p53, or Ad/Bax at an MOI of 500024 hours later. Cell viability was assessed 24, 48, and 72 hours after adenoviral infection using a colorimetric assay with tetrazolium blue dye 2,3 -bis-(2-methoxy-4-nitro-5sulphenyl)-(2-H)-tetrazolium-5-carboxamide (XTT) and the Cell Proliferation Kit II (Roche Molecular Biochemicals, Indianapolis, Indiana, USA) according to the manufacturer's protocol. ${ }^{19}$ The experiments were performed at least twice for each cell line. To examine a dose dependent effect of Ad/Bax, MKN-45 cells were infected with increasing MOIs of Ad/Bax and cell viability was assessed 72 hours after viral infection.

\section{Hoechst staining}

Cells were plated in 12 well plates at a density of $3 \times 10^{4}$ cells/ well and infected with Ad/LacZ or Ad/Bax at an MOI of 5000 24 hours later. Forty eight hours after infection, cells were fixed in $2 \%$ glutaraldehyde for two hours and stained with Hoechst 33258.

\section{Western blot analysis}

Cells were placed in a $10 \mathrm{~cm}$ tissue culture dish and infected with each virus at an MOI of 5000 for 72 hours. Cells were then washed with ice cold phosphate buffered saline (PBS) and lysed with the addition of $100 \mu \mathrm{l}$ of $2 \times$ sodium dodecyl sulphate (SDS) buffer. Protein concentration of the total cell lysates was determined using a protein assay kit (Bio-Rad, Melville, New York, USA). Lysates (40 $\mu \mathrm{g} / \mathrm{lane})$ were separated on $10 \%$ SDS-polyacrylamide gel electrophoresis (PAGE) gels and then electrophoretically transferred to nitrocellulose membranes at $15 \mathrm{~V}$ for 30 minutes. After blocking with $5 \%$ bovine serum albumin in washing buffer containing $140 \mathrm{mM} \mathrm{NaCl}, 25 \mathrm{mM}$ Tris $\mathrm{HCl} \mathrm{pH} \mathrm{7.8,} \mathrm{and}$ $0.05 \%$ Tween 20 , membranes were incubated with primary antibodies overnight at $4^{\circ} \mathrm{C}$ and thereafter incubated with the corresponding peroxidase linked secondary antibodies at $37^{\circ} \mathrm{C}$ for one hour. Signals were developed using a standard enhanced chemiluminescence method following the manufacturer's protocol (Amersham Bioscience, Piscataway, New Jersey, USA).

\section{Animal experiments}

Human gastric cancer MKN-45 xenografts were established in six week old female nude mice (Balb/c nu/nu) through subcutaneous inoculation of $2 \times 10^{6} \mathrm{MKN}-45$ cells into the dorsal flank. Each tumour reached a diameter of approximately 3-5 mm six days after inoculation; each mouse was then given an intratumoral injection of $100 \mu \mathrm{l}$ of $2 \times 10^{10} \mathrm{vp}$ of Ad/LacZ, Ad/CMV-p53, or Ad/Bax. Tumours were measured 2-3 a week, and tumour volume was calculated using the equation $a \times b^{2} \times 0.5$, in which $a$ and $b$ are the largest and smallest diameters, respectively. Animals were killed when their tumour reached a diameter of approximately $15 \mathrm{~mm}$. To develop peritoneally disseminated xenografts of human gastric cancer, mice were inoculated with $2 \times 10^{6} \mathrm{MKN}-45$ cells into the peritoneal cavity. Also, to assess the efficiency of adenoviral gene transfer into the peritoneally disseminated tumours, mice were injected with $200 \mu \mathrm{l}$ of $3 \times 10^{10} \mathrm{vp}$ of $\mathrm{Ad} / \mathrm{LacZ}$ into the peritoneal cavity 26 days after inoculation of MKN-45 cells. These mice were killed 48 hours later, and $\mathrm{X}$-gal staining was performed. To assess the antitumour effect of the viruses, mice were given an intraperitoneal injection of $200 \mu \mathrm{l}$ of $2 \times 10^{10} \mathrm{vp}$ of each virus six days after inoculation of MKN-45 cells. Mice were killed 24 days after injection, and all visible disseminated tumours were counted and weighed. The survival study was also performed for all four groups.

\section{Statistics}

Statistical analysis was performed using the Student's $t$ test with the statistical software program (DA Stats).

\section{RESULTS}

\section{Adenovirus mediated gene transfer into gastric cancer} cell lines

The efficiency of adenovirus mediated gene transfer into gastric cancer cell lines was assessed using X-gal staining 48 hours after infection with $\mathrm{Ad} / \mathrm{LacZ}$ with a binary adenoviral vector system. Ad/LacZ transferred the LacZ gene into these cell lines. An MOI of 5000 was chosen for further

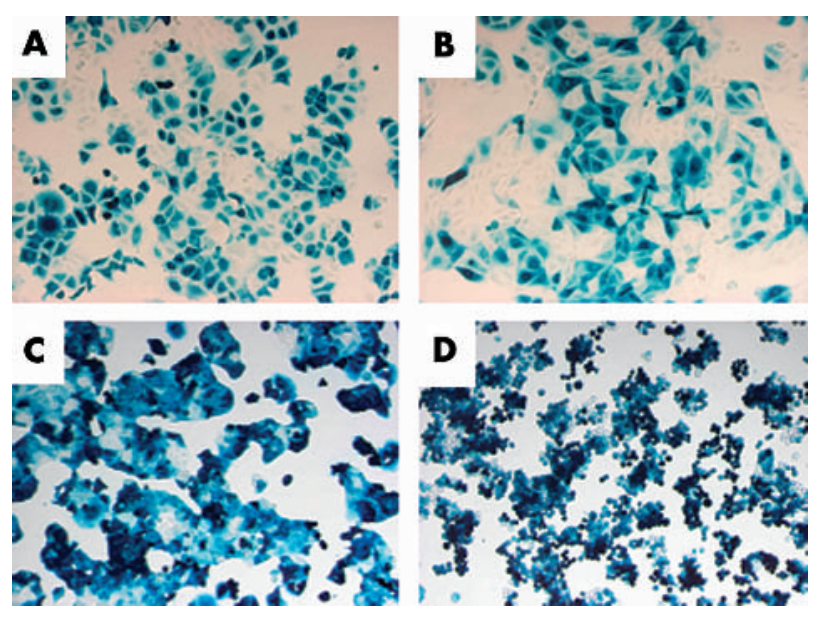

Figure 1 Transduction efficiency of $\mathrm{Ad} / \mathrm{LacZ}$ in gastric cancer cells. (A) MKN-1, (B) MKN-7, (C) MKN-28, and (D) MKN-45 cells were plated on 24 well plates and infected with $\mathrm{Ad} / \mathrm{LacZ}$ at a multiplicity of infection of 5000 . After 48 hours, cells were fixed and stained with 5-bromo-4-chloro-3-indolyl- $\beta$-D-galactopyranoside (X-gal) to demonstrate LacZ gene expression. Original magnification, $\times 100$. 
in vitro study because it provided maximum transduction efficacy and minimal intrinsic toxicity in these cell lines. More than $90 \%$ of $\mathrm{MKN}-1$ (as per fig 1), MKN-28, and MKN-45 cells and $55.2 \%$ of MKN-7 cells stained positively for X-gal (fig 1). These data demonstrated that a binary adenoviral vector system is capable of efficient transgene expression in human gastric cancer cell lines.

\section{Inhibition of cell growth in vitro}

To evaluate the antitumour effect of the Bax gene in gastric cancer cells, cells were treated with Ad/Bax. Cell viability was determined using the XTT assay 24, 48, and 72 hours after infection. The resulting data were compared with those of treatment with $\mathrm{Ad} / \mathrm{p} 53$. Treatment with both $\mathrm{Ad} / \mathrm{Bax}$ and $\mathrm{Ad} /$ p53 significantly suppressed the growth of MKN-1, MKN-7,
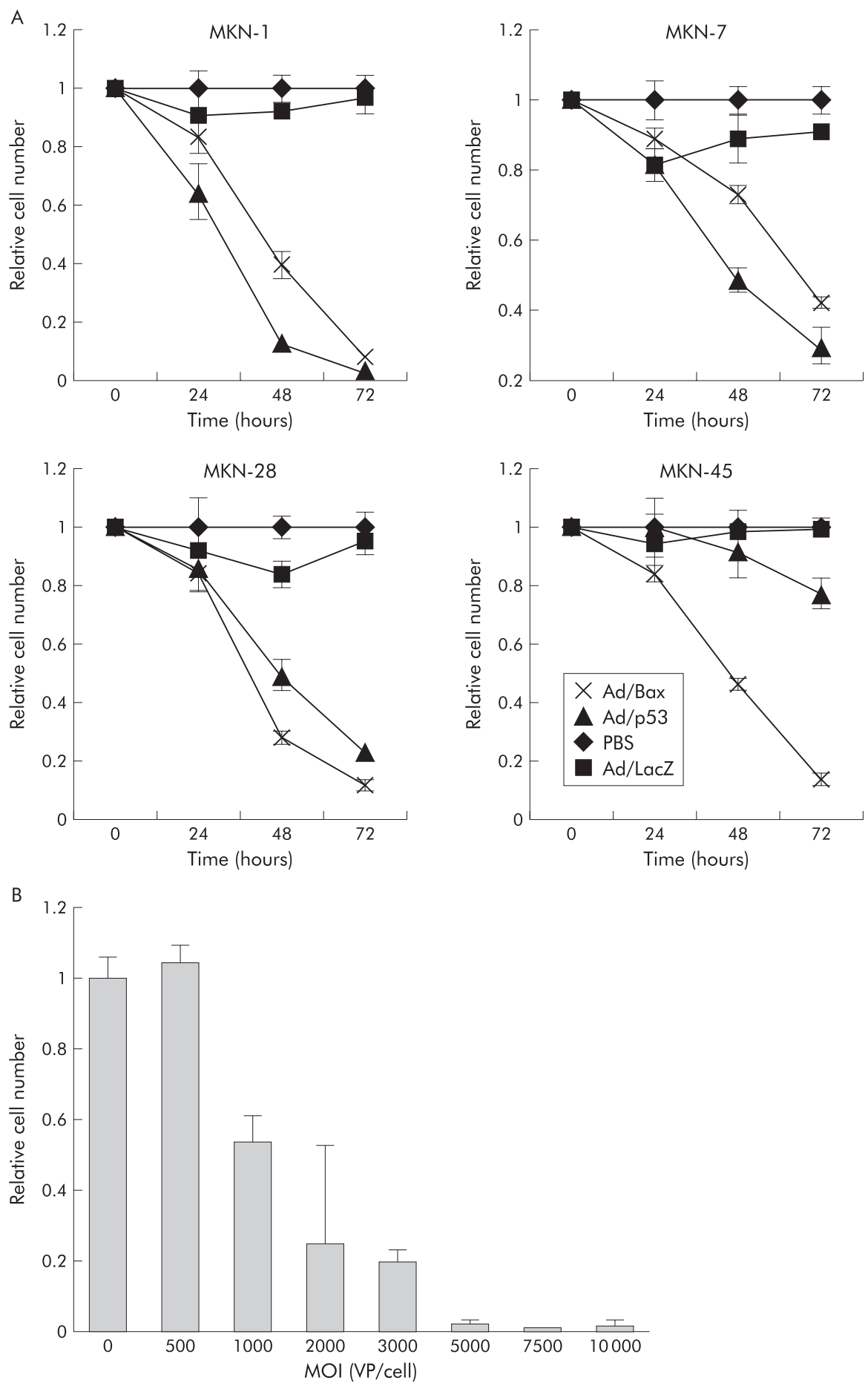

Figure 2 (A) Cell viability, as determined using a colorimetric assay with 2,3-bis-(2-methoxy-4-nitro-5-sulphenyl)-(2H)-tetrazolium-5-carboxanilide (XTT), after infection. The viability value was expressed relative to that of cells infected with phosphate buffered saline (PBS), which was arbitrarily referred to as 1 . Values for the Ad/Bax and Ad/p53 groups were significantly different from those for the PBS and Ad/LacZ groups in MKN-1, $M K N-7$, and MKN-28 whereas only treatment with Ad/Bax significantly differed from that in the other groups in MKN-45 cells. Values are means (SD) of one of two similar quadruplicate studies for each groups. (B) MKN-45 cells were mock infected or infected with increasing multiplicities of infection (MOls) of $\mathrm{Ad} / \mathrm{Bax}$ and then subjected to the XTT assay 72 hours after viral infection. Values are mean (SD). VP, viral particles. 


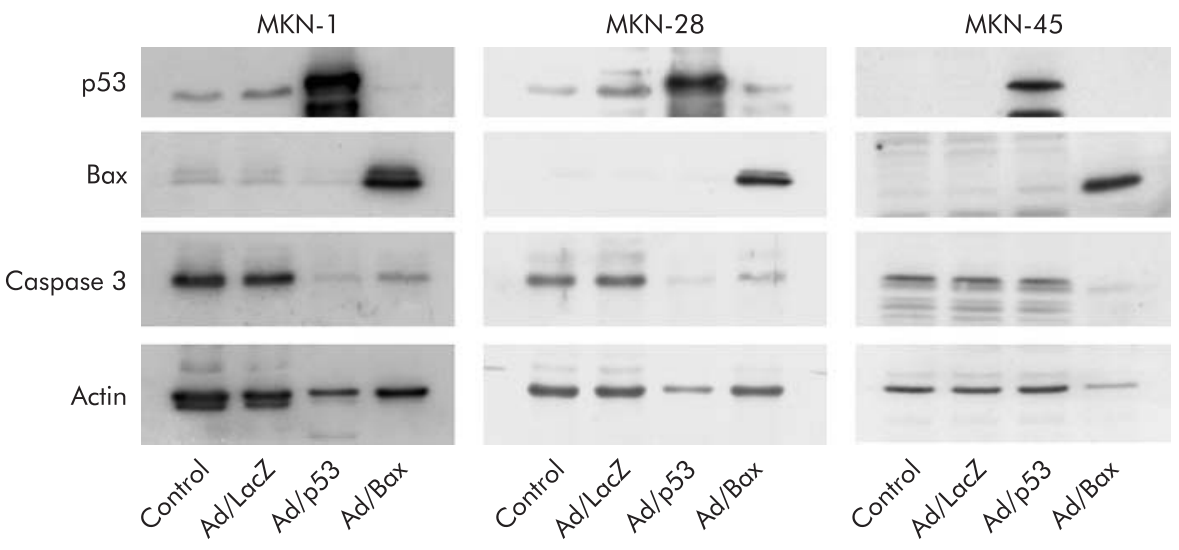

Figure 3 Adenovirus mediated p53, Bax, and caspase 3 protein expression in the human gastric cancer cell lines MKN-1, MKN-28, and MKN-45. The cell lines were infected with $\mathrm{Ad} / \mathrm{LacZ}, \mathrm{Ad} / \mathrm{p} 53$, or Ad/Bax at a multiplicity of infection of 5000 . Cell lysates were harvested 72 hours after infection and subjected to $10 \%$ sodium dodecyl sulphate-polyacrylamide gel electrophoresis and western blot analysis using the specified antibodies. The molecular weights of $\mathrm{Bax}, \mathrm{p} 53$, caspase 3 , and actin were $21 \mathrm{kDa}, 53 \mathrm{kDa}, 32 \mathrm{kDa}$, and $43 \mathrm{kDa}$, respectively.

and MKN-28 cells. However, only treatment with Ad/Bax significantly suppressed cell viability in MKN-45 cells (fig 2A); treatment with Ad/p53 was not effective. Infection with Ad/Bax produced a dose dependent inhibition of tumour growth in MKN-45 cells (fig 2B). The gastric cancer cell lines that retained wild-type $p 53$ expression, such as MKN-45, appeared to be resistant to $p 53$ treatment.

\section{Gene expression and induction of apoptosis}

Expression of Bax, p53, and caspase 3 protein in MKN-1, MKN-28, and MKN-45 cells was detected using western blot analysis. Weak expression of Bax protein was detected in all three human gastric cancer cell lines without $\mathrm{Ad} / \mathrm{Bax}$ infection (fig 3). As the most frequent abnormality of the Bax gene is a frameshift mutation that causes truncated undetectable Bax protein, ${ }^{20}$ the result revealed that these gastric cancer cell lines contained the wild-type Bax gene. A high level of expression of Bax and p53 protein was observed in these cell lines when they were infected with $\mathrm{Ad} / \mathrm{Bax}$ and Ad/p53, respectively, at an MOI of 5000 for 72 hours (fig 3). When MKN-1 and MKN-28 cells were treated with either
$\mathrm{Ad} / \mathrm{Bax}$ or $\mathrm{Ad} / \mathrm{p} 53$, procaspase 3 protein expression was diminished, which means that caspase 3 was activated by cleavage. In MKN-45 cells, activation of caspase 3 was observed only with treatment with $\mathrm{Ad} / \mathrm{Bax}$ and not Ad/p53 (fig 3). On Hoechst staining, both $\mathrm{Ad} / \mathrm{p} 53$ and $\mathrm{Ad} / \mathrm{Bax}$ treatment resulted in an apoptotic morphology with features such as condensation of chromatin in MKN-1 and MKN-28. This morphology was observed only in Ad/Bax treated MKN45 cells however (fig 4). These data demonstrated that $\mathrm{Ad} /$ p53 treatment induced apoptosis by activating the caspase cascade in MKN-1 and MKN-28, but not in MKN-45, cells. Only $\mathrm{Ad} / \mathrm{Bax}$ was effective in triggering apoptosis even in MKN-45 cells that were refractory to $\mathrm{Ad} / \mathrm{p} 53$.

\section{Ad/Bax suppressed subcutaneous MKN-45 tumour growth}

The efficacy of Ad/Bax treatment of subcutaneous tumours derived from $\mathrm{Ad} / \mathrm{p} 53$ resistant MKN-45 cells was further assessed. Each virus was injected directly into the established tumours, and eight animals per group were monitored for changes in tumour size after injection. Only treatment with

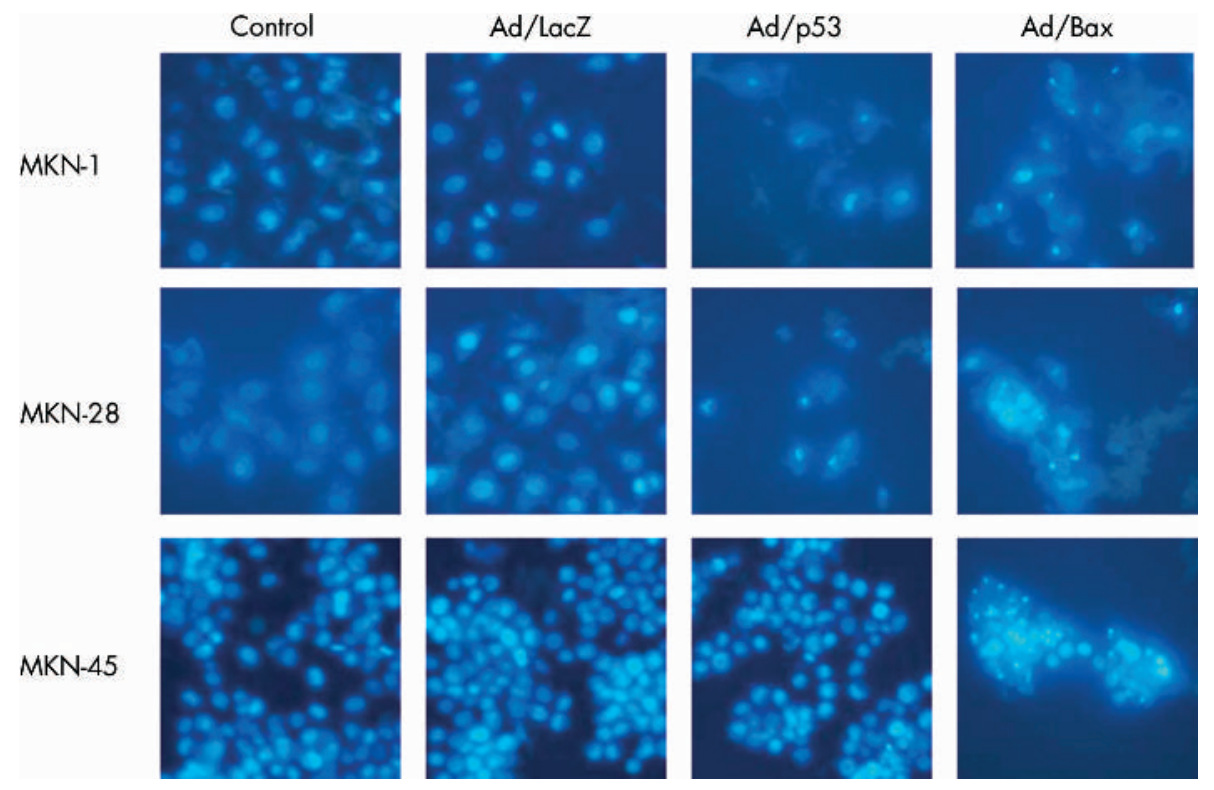

Figure 4 Hoechst staining for apoptosis. Condensation of chromatin was observed in MKN-1 and MKN-28 cells infected with $\mathrm{Ad} / \mathrm{p} 53$ or $\mathrm{Ad} / \mathrm{Bax}$ at a multiplicity of infection (MOI) of 5000 for 48 hours. In MKN-45 cells, infection with only Ad/Bax was observed at an $\mathrm{MOI}$ of 5000 for 48 hours. 
Ad/Bax significantly suppressed tumour growth compared with controls. Mean tumour volume in the Ad/Bax group was only $236.5(83.8) \mathrm{mm}^{3}$ whereas that of the $\mathrm{Ad} / \mathrm{p} 53$ group was $515.2(151.9) \mathrm{mm}^{3}, 35$ days after inoculation $(\mathrm{p}<0.01)$. Although treatment with $\mathrm{Ad} / \mathrm{p} 53$ suppressed tumour growth compared with no treatment $(\mathrm{p}<0.05)$, the difference compared with Ad/LacZ treatment was not significant (fig 5).

\section{Inhibition of peritoneal dissemination by intraperitoneal injection of $\mathrm{Ad} / \mathrm{Bax}$}

One of the best measures of the extent of gastric cancer is peritoneal dissemination. To further assess the efficacy of Bax gene transfer, peritoneal disseminated $\mathrm{MKN}-45$ tumours were generated in nude mice. To examine transgene expression by a binary vector system in vivo, $\mathrm{Ad} / \mathrm{LacZ}$ was injected into the peritoneal cavity of the mice; their organs were subjected to X-gal staining 48 hours later. The LacZ gene was transferred mainly into the superficial layers of the peritoneal tumours and not normal tissue (fig 6A). This was consistent with the expectation that deeper levels of an implanted tumour may not be infected by an adenovirus, administered when the macroscopic tumour was established. Unexpectedly, transgene expression was relatively selective to the tumour surface and few of the peritoneal cells stained blue. This may be due to differences in susceptibility to adenoviral infection between the implanted tumour and surrounding normal peritoneum. These data showed that a binary adenoviral vector system is capable of transferring the $\mathrm{LacZ}$ gene to a tumour via intraperitoneal administration.

The efficacy of Ad/Bax treatment was then assessed using the same MKN-45 peritoneally disseminated model. Ad/LacZ, $\mathrm{Ad} / \mathrm{p} 53$, or $\mathrm{Ad} / \mathrm{Bax}$ was injected into the peritoneal cavity six days after inoculation when the tumour was microscopic to maximise the efficiency of adenoviral gene transfer. Both the total number and weight of the intraperitoneal tumours were assessed 24 days after treatment in each group. The mean total number in the Ad/p53 group was 14.75 (2.44) while that in the $\mathrm{Ad} / \mathrm{Bax}$ was 5.5 (3.32). Also, the mean total tumour weight in the Ad/p53 group was 371.8 (44.0) $\mu \mathrm{g}$ while that in the Ad/Bax group was 161.9 (96.9) $\mu \mathrm{g}$, demonstrating that $\mathrm{Ad} / \mathrm{Bax}$ treatment significantly suppressed intraperitoneal tumour growth $(\mathrm{p}<0.01)$. Although the difference in tumour number and weight between the Ad/p53 and no treatment groups was significant $(\mathrm{p}<0.01)$, that between the $\mathrm{Ad} / \mathrm{p} 53$ and $\mathrm{Ad} /$ LacZ groups was not significant (fig 6B, C). Survival curves for all four groups are shown in fig 6D. Control mice without treatment all died within 42 days of tumour inoculation. Only three of eight mice treated with Ad/p53 survived for 60 days after tumour inoculation whereas six of eight mice treated with Ad/Bax survived.

\section{DISCUSSION}

Cancer can be attributed in part to defects in the regulation of apoptotic cell death. Failure to trigger apoptosis also renders cancers resistant to anticancer therapy. Recent studies of regulation of apoptosis at the molecular level have raised expectations of development of effective apoptosis targeting therapies such as gene therapy. Gene therapy has been explored as a new strategy against various cancers; it has even been studied in clinical trials. ${ }^{23}$ Adenovirus mediated p53 gene transfer has been extensively studied to evaluate its clinical application in gene therapy for various cancers. ${ }^{5-11}$ Several groups have reported on the use of experimental gene therapy against gastric cancers using the $p 53$ gene. Tatebe et al examined the effect of a $p 53$ adenoviral vector on gastric cancer cell lines in vitro. They found that some of the cell lines were resistant to this $p 53$ treatment. These authors speculated that the difference in treatment outcome may have been due to different bcl-2 family protein statuses. ${ }^{9}$
Also, Ohashi et al reported that an adenovirus carrying $p 53$ effectively inhibited the growth of $p 53$ mutant gastric tumours in mice. ${ }^{10}$ However, it failed to suppress the growth of MKN-45 cells carrying wild type $p 53$. This finding is consistent with reports by others showing that cancer cells carrying wild-type $p 53$ are relatively resistant to treatment with the $p 53$ gene. ${ }^{12}{ }^{13}$ The Bax gene may serve as a good alternative to $p 53$ for gene therapy for cancer, not only because it may kill cancer cells directly but also because it may increase their sensitivity to other antitumour treatments. Researchers have shown that Bax overexpression enhances apoptosis after treatment with chemotherapeutic agents in human breast cancer cells ${ }^{21}$ and ovarian cancer cells, ${ }^{22}$ and after radiotherapy in human breast cancer cells. ${ }^{23}$ Based on this evidence, Bax gene therapy may improve on current conventional cancer therapy.

Our previous studies demonstrated that overexpression of Bax protein in a binary adenoviral mediated system can express Bax protein and induce apoptosis in vivo in lung and prostate cancer models. ${ }^{132425}$ In the present study, we demonstrated that Bax gene transfer effectively induced apoptosis in gastric cancer cells and suppressed gastric tumour growth both in vitro and in vivo. Furthermore, we demonstrated that adenovirus mediated Bax gene transfer effectively killed even $p 53$ resistant gastric cancer cells.

Recent preclinical studies of disseminated gastric cancer included intraperitoneal injection of a fusogenic liposome containing a mutated degenerin gene ${ }^{26}$ and cisplatin encapsulated by a transferring conjugated liposome. ${ }^{27}$ To evaluate the antitumour effect of the Bax gene on peritoneal gastric tumours, we performed intraperitoneal injection of the adenoviral vector Ad/Bax. Our results showed that injection of $\mathrm{Ad} / \mathrm{Bax}$ suppressed the growth of disseminated gastric cancer in the peritoneal cavity and prolonged the survival of mice. On staining of visceral organs and tumours with X-gal, we found that the gene of interest had been transferred to the surface of the peritoneal tumours, although it appeared to be difficult to deliver the gene into the deeper layers of the tumours via intraperitoneal injection of the adenoviral

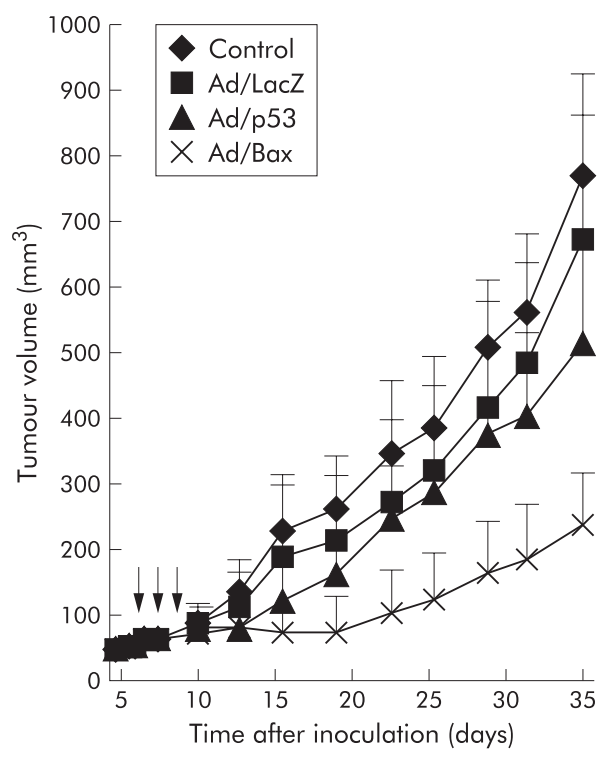

Figure 5 Suppression of the growth of subcutaneous tumours derived from MKN-45 cells treated with a control (no treatment), Ad/LacZ, $\mathrm{Ad} / \mathrm{p} 53$, or $\mathrm{Ad} / \mathrm{Bax}$ by adenovirus mediated gene transfer. Tumour volume was monitored over time (days) after inoculation of tumour cells. Arrows, time points where treatment was given (days 6, 7, and 8). Values represent the means (SD) of eight mice per group. Treatment with $\mathrm{Ad} / \mathrm{Bax}$ differed significantly from that in the other groups $(p<0.01)$. 

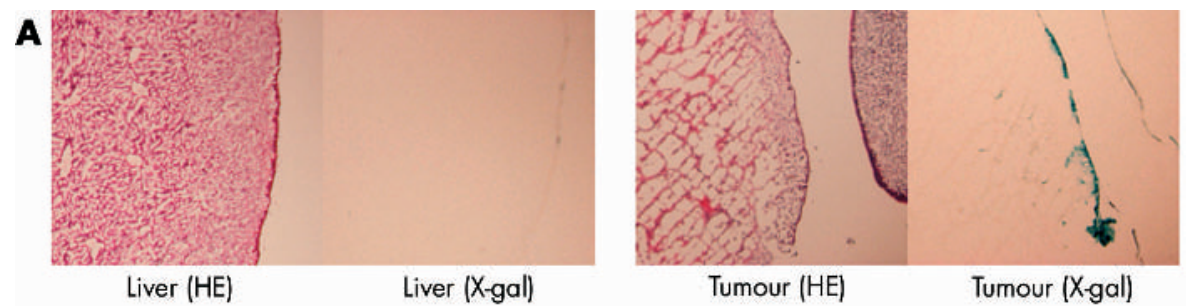

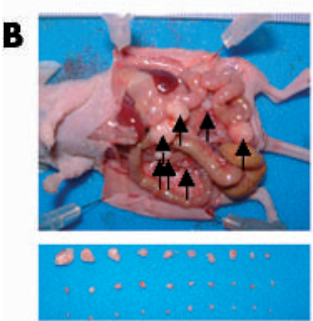

Control
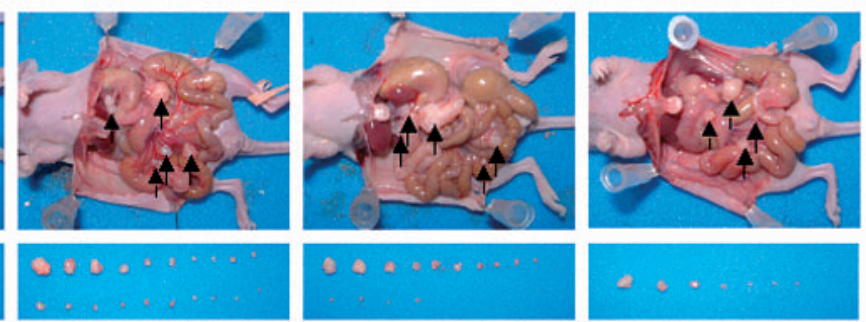

$\mathrm{Ad} / \operatorname{LacZ}$

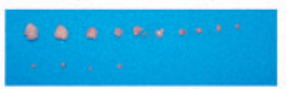

$\mathrm{Ad} / \mathrm{p} 53$

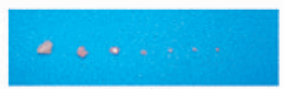

$\mathrm{Ad} / \mathrm{Bax}$
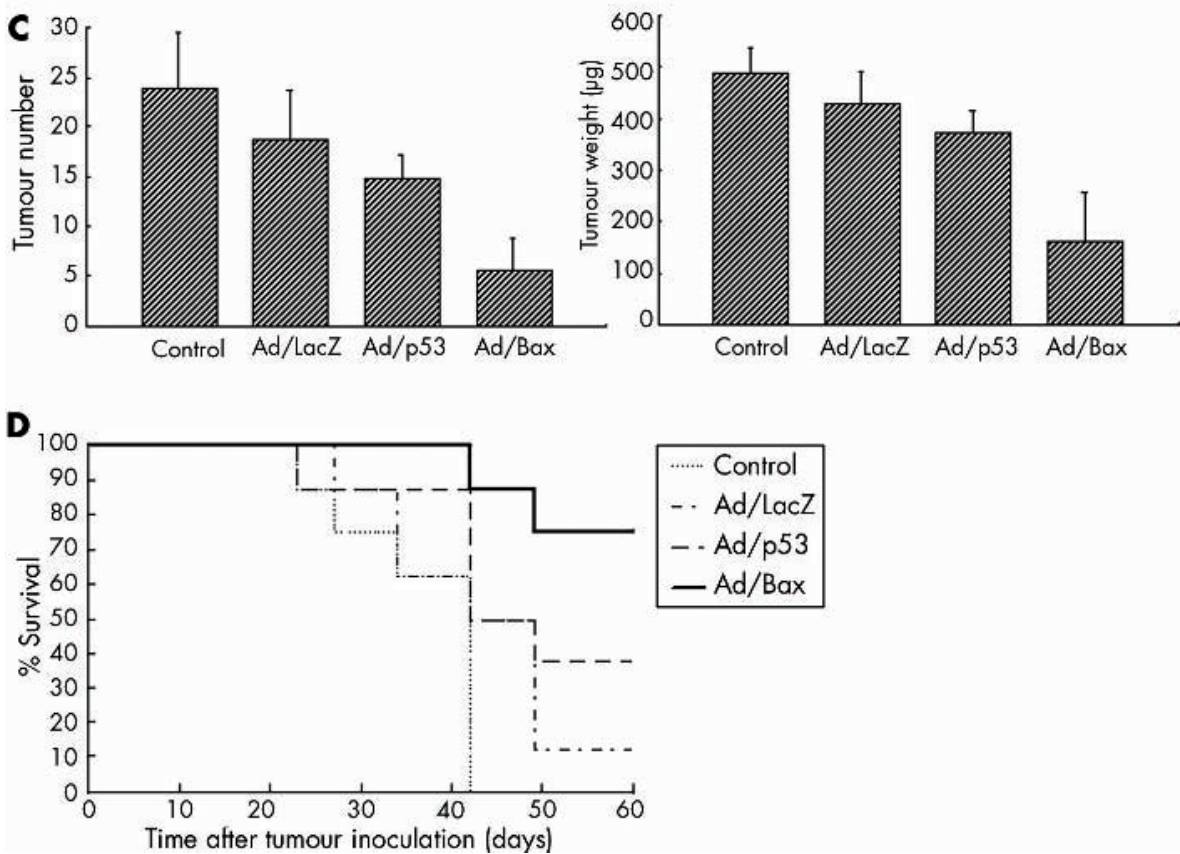

Figure 6 (A) Transduction efficiency of Ad/LacZ in MKN-45 cells in vivo. Twenty six days after tumour cell inoculation, mice were injected with $\mathrm{Ad} /$ LacZ at $3 \times 10^{10}$ particles into the peritoneal cavity. Forty eight hours after inoculation, mice were killed and 5-bromo-4-chloro-3-indolyl- $\beta$-Dgalactopyranoside (X-gal) staining was performed. Original magnification, $\times 40$. (B, C) On days 6,7 , and 8 after inoculation, mice received no treatment (Control), Ad/LacZ, Ad/p53, or Ad/Bax at $2 \times 10^{10}$ particles. (B) Macroscopic appearance of peritoneal dissemination after treatment on day 24 after inoculation. Arrows, disseminated tumour. (C) Total number (left) and weight (right) of disseminated tumours. Values represent the means (SD) of eight mice per group. Treatment with $\mathrm{Ad} /$ Bax differed significantly from that in the other groups $(p<0.01)$. (D) Survival of mice bearing peritoneal dissemination of $M K N-45$ tumours treated with intraperitoneal injection of $\mathrm{Ad} /$ Bax. Survival was monitored over time after tumour injection and plotted as a Kaplan-Meier plot.

vectors. Therefore, we began treatment only six days after intraperitoneal inoculation of $\mathrm{MKN}-45$ cells when the tumours were only microscopic nodules. This experiment may mimic the postoperative peritoneal dissemination of gastric cancer without other clinical or radiological evidence of the disease, supposing the use of adjuvant therapy. Unlike intratumoral injection of adenoviral vectors, intraperitoneal injection results in dilution of the virus solution in the peritoneal cavity and reduction of transduction efficacy. Therefore, use of a more potent gene than $p 53$ that can accomplish this purpose with lower transgene copy numbers is required. Based on this viewpoint, the Bax gene is more suitable than the $p 53$ gene for inducing apoptosis of cancer cells. Theoretically, Bax gene transfer with a replication incompetent adenovirus can kill only transduced cells. Use of a replication competent adenovirus or in combination with another modality such as chemotherapy or radiotherapy may improve treatment outcomes.

As it is a strong proapoptotic gene, overexpression of Bax may also induce apoptosis of normal cells. In fact, we previously demonstrated that intravenous infusion of a Bax expressing adenoviral vector induced rapid massive apoptosis of hepatocytes in mice. ${ }^{16}$ However, there was very little Bax expression in normal organs after intraperitoneal injection of the adenovirus, and macroscopic examination showed that the liver of mice treated with Ad/Bax was similar to that in mice treated with other vectors. Reports by others suggested that systemic absorption of an adenovirus from the peritoneal cavity sometimes occurred after intraperitoneal injection of the adenovirus. ${ }^{28}$ 
Intraperitoneal injection of an adenovirus once a week showed no abnormalities on biochemistry tests and haematoxylin and eosin staining of sections of the various organs. ${ }^{29}$ In the present study, macroscopic examination showed that the liver of mice treated with an adenovirus was slightly swollen, independent of the gene transferred. Although we tested only one dosing schedule of three sequential intraperitoneal injections in this study, other schedules may reduce the toxicity.

In conclusion, application of $\mathrm{Ad} / \mathrm{Bax}$ may be useful for treating gastric cancer cells in spite of the status of the $p 53$ gene.

\section{ACKNOWLEDGEMENTS}

This work was supported in part by grants from the Ministry of Education, Science, and Culture, Japan; grants from the Ministry of Health and Welfare, Japan (Health Sciences Research Grants (Research on Human Genome and Gene Therapy)); a grant from the National Cancer Institute and National Institutes of Health (POI CA78778) to (JAR); a SPORE grant (2P50 CA70907) to (JAR); and the WM Keck Foundation (JAR). SK is supported by Research Fellowships from the Japan Society for the Promotion of Science for Young Scientists. We thank Yoshiko Shirakiya for technical assistance and Alma Vega for her expert assistance in the preparation of this manuscript.

\section{Authors' affiliations}

Y Tsunemitsu, N Tokunaga, S Otani, T Umeoka, N Tanaka, Division of Surgical Oncology, Department of Surgery, Okayama University Graduate School of Medicine and Dentistry, Okayama 700-8558, Japan

S Kagawa, T Fujiwara, Division of Surgical Oncology, Department of Surgery, Okayama University Graduate School of Medicine and Dentistry, Okayama 700-8558, Japan, and Centre for Gene and Cell Therapy, Okayama University Hospital, Okayama 700-8558, Japan J A Roth, B Fang, Department of Thoracic and Cardiovascular Surgery, University of Texas MD. Anderson Cancer Center, Houston, TX 77030, USA

\section{REFERENCES}

1 Wanebo HJ, Kennedy BJ, Chmie J, et al. Cancer of the stomach. A patient care study by the American Collage of Surgeons. Ann Surg 1993;218:583-92.

2 Roth JA, Nguyen D, Lawrence DD, et al. Retrovirus-mediated wild-type p53 gene transfer to tumors of patients with lung cancer. Nat Med 1996;2:985-91.

3 Swisher SG, Roth JA, Nemunaitis J, et al. Adenovirus-mediated p53 gene transfer in advanced non-small-cell lung cancer. J Natl Cancer Inst 1999;91:763-71.

4 Levine AJ. p53, the cellular gatekeeper for growth and division. Cell 1997;88:323-31.

5 Fujiwara T, Grimm EA, Mukhopadhyay T, et al. Induction of chemosensitivity in human lung cancer cells in vivo by adenovirus-mediated transfer of the wildtype p53 gene. Cancer Res 1994;54:2287-91.

6 Spitz FR, Nguyen D, Skibber JM, et al. In vivo adenovirus-mediated p53 tumor suppressor gene therapy for colorectal cancer. Anticancer Res $1996 ; 16: 3415-22$.
7 Bouvet M, Bold RJ, Lee J, et al. Adenovirus-mediated wild-type p53 tumor suppressor gene therapy induces apoptosis and suppresses growth of human pancreatic cancer. Ann Surg Oncol 1998;5:681-8.

8 Nielsen LL, Lipari P, Dell J, et al. Adenovirus-mediated p53 gene therapy and paclitaxel have synergistic efficacy in models of human head and neck, ovarian, prostate, and breast cancer. Clin Cancer Res 1998;4:835-46.

9 Tatebe S, Matsuura T, Endo K, et al. Adenovirus-mediated transfer of wildtype p53 gene results in apoptosis or growth arrest in human cultured gastric cancer cells. Int J Oncol 1999;15:229-35.

10 Ohashi M, Kanai F, Ueno H, et al. Adenovirus mediated p53 tumor suppressor gene therapy for human gastric cancer cells in vitro and in vivo. Gut 1999;44:366-71.

11 Shimada H, Shimizu T, Ochiai T, et al. Preclinical study of adenoviral p53 gene therapy for esophageal cancer. Surg Today 2001;31:597-604.

12 Harris MP, Sutiipto S, Wills KN, et al. Adenovirus-mediated p53 gene transfer inhibits growth of human tumor cells expressing mutant p53 protein. Cancer Gene Ther 1996;3:121-30.

13 Kagawa S, Gu J, Swisher SG, et al. Antitumor effect of adenovirus-mediated Bax gene transfer on p53-sensitive and p53-resistant cancer lines. Cancer Res 2000;60:1157-61.

14 Jurgensmeier JM, Xie Z, Deveraux $Q$, et al. Bax directly induces release of cytochrome c from isolated mitochondria. Proc Natl Acad Sci U S A 1998;95:4997-5002.

15 Pastorino JG, Chen ST, Tafani M, et al. The overexpression of Bax produces cell death upon induction of the mitochondrial permeability transition. J Biol Chem 1998;273:7770-5.

16 Kagawa S, Pearson SA, Ji L, et al. A binary adenoviral vector for expressing high levels of the proapoptotic gene bax. Gene Ther 2000;7:75-9.

$17 \mathrm{Ji} \mathrm{L}$, Bouvet M, Price RE, et al. Reduced toxicity, attenuated immunogenicity and efficient mediation of human p53 gene expression in vivo by an adenovirus vector with deleted E1, E3 and inactivated E4 by GAL4-TATA promoter replacement. Gene Ther 1999;6:393-402.

18 Fang B, Ji L, Bouvet M, et al. Evaluation of GAL4/TATA in vivo. Induction of transgene expression by adenovirally mediated gene codelivery. J Biol Chem 1998;273:4972-5.

19 Roehm NW, Rodgers GH, Hatfield SM, et al. An improved colorimetric assay for cell proliferation and viability utilizing the tetrazolium salt XTT. J Immunol Methods 1991;142:257-65.

20 Mrozek A, Petrowsky H, Sturm I, et al. Combined p53/Bax mutation results in extremely poor prognosis in gastric carcinoma with low microsatellite instability. Cell Death Differ 2003;10:461-7.

21 Wagener C, Bargou RC, Daniel PT, et al. Antitumor activity of bax and p53 naked gene bax- $\alpha$ sensitizes cultured breast cancer cells to drug-induced apoptosis. Int J Cancer 1996;67:138-41.

22 Strobel T, Swanson L, Korsmeyer T, et al. BAX enhances paclitaxel-induced apoptosis through a p53-independent pathway. Proc Natl Acad Sci U S A 1996;93:14094-9.

23 Sakakura C, Sweeney EA, Shirahama T, et al. Overexpression of bax sensitizes human breast cancer MCF-7 cells to radiation-induced apoptosis. Int J Cancer 1996:67:101-5.

24 Li X, Marani M, Yu J, et al. Adenovirus-mediated Bax overexpression for the induction of therapeutic apoptosis in prostate cancer. Cancer Res 2001;61:186-91.

25 Honda T, Kagawa S, Spurgers KB, et al. A recombinant adenovirus expressing wild-type Bax induces apoptosis in prostate cancer cells independently of their $\mathrm{Bcl}-2$ status and androgen sensitivity. Cancer Biol Ther 2002;1:163-7.

26 Horimoto M, Sasaki Y, Ugawa S, et al. A novel strategy for cancer therapy by mutated mammalian degenerin gene transfer. Cancer Gene Ther 2000;7:1341-7.

27 linuma H, Maruyama K, Okinaga K, et al. Intracellular targeting therapy of cisplatin-encapsulated transferrin-polyethylene glycol liposome on peritoneal dissemination of gastric cancer. Int J Cancer 2002;99:130-7.

28 Grace MJ, Xie L, Musco ML, et al. The use of laser scanning cytometry to assess depth of penetration of adenovirus p53 gene therapy in human xenograft biopsies. Am J Pathol 1999;155:1869-78.

29 Saimura M, Nagai E, Mizumoto K, et al. Intraperitoneal injection of adenovirus-mediated NK4 gene suppresses peritoneal dissemination of pancreatic cancer cell line AsPC-1 in nude mice. Cancer Gene Ther 2002;9:799-806. 